\title{
In-plane and out-of-plane MEMS gyroscopes based on piezoresistive NEMS detection
}

\author{
Stefano Dellea, Federico Giacci, Antonio Francesco Longoni, and Giacomo Langfelder
}

\begin{abstract}
The work presents a new design and a complete characterization of AM gyroscopes based on piezoresistive nanogauges. The working principle and optimization criteria of inplane and out-of-plane devices, relying on double frame decoupling and levered sense mode, are discussed in light of sensitivity and resolution theoretical predictions. The architecture of driving and sensing electronics is also presented. The reduced thermo-mechanical damping with respect to capacitive configurations, and the inherently high output signal lead to white noise performance in the mdps $/ \sqrt{ } \mathrm{Hz}$ range within an area smaller than $0.35 \mathrm{~mm}^{2}$, at pressures in the mbar range. Sub5-ppm linearity errors within $1000 \mathrm{dps}$ are also demonstrated.
\end{abstract}

Index Terms - MEMS gyroscopes, NEMS gauges, inertial sensors, piezoresistive sensing, motion amplitude control.

\section{INTRODUCTION}

$\mathrm{T}$ HE consumer market still demands for miniaturization, power consumption reduction, and performance improvement of microelectromechanical system (MEMS) gyroscopes [1-3]. Optical image stabilization capabilities drive the requirements on white noise [4]. Navigation drives the requirements on long-term offset drift (bias stability) [5].

In this context, most of MEMS gyroscopes are based on capacitive measurement of the angular rate through the Coriolis force occurring (along the sense direction) on a suspended mass kept in motion with a controlled amplitude along the drive direction [6-8]. This operation is referred to as amplitude-modulation (AM) mode. In mode-matched AM operation (same frequencies of drive and sense modes), the gyroscope gain is the largest one, but it is difficult to simultaneously reach low-noise, large system bandwidth and long-term stability at low power consumption [9, 10]. AM gyroscopes are thus commonly operated in mode-split conditions, i.e. with an intended offset between the drive and sense frequencies. Advantages of improved stability and extended bandwidth come at the cost of a reduced device gain [11], which makes the white noise density limited by the electronics to sub-10 mdps $/ \sqrt{ } \mathrm{Hz}$ in typical products [11-15].

Recently, to overcome this inherent drawback of mode-split operation, alternative working principles were proposed, based on frequency modulation (FM). Quadrature FM (QFM) gyroscopes detect the angular rate through the frequency variation in a mode-matched device where drive and sense

The authors are with the Department of Electronics, Information Technology and Bioengineering, Politecnico di Milano, Milano, 20133, Italy e-mail: giacomo.langfelder@polimi.it. modes are kept in quadrature oscillation [16]. The QFM gyroscopes stability is limited by temperature effects on the non-natively-matched modes. White noise is limited by supply noise (which turns into frequency noise via spring softening). Lissajous FM (LFM) gyroscopes [17] bypass the offset issue by continuously modulating the relative phase between the modes, and in turn the sensitivity. In other words, LFM gyroscopes need again a split by an intended mismatch. As a consequence, the obtainable signal-to-white-noise ratio has the same expression as in mode-split AM gyroscopes [18]. For both QFM and LFM devices, the required axisymmetric structure makes the design of $\mathrm{X}$ - and $\mathrm{Y}$-axis gyroscopes quite challenging. Only Z-axis structures were indeed shown so far.

An alternative approach to reduce the impact of electronic noise is to exploit sensing principles different from capacitive sensing in AM gyroscopes. In this work, gyroscopes based on nanoelectromechanical system (NEMS) piezoresistive sensing elements are presented. At gauge factors in the order of 50 [19-20], NEMS gauges have an inherent advantage of giving a large output signal. This consistently reduces the impact of electronics noise. Therefore, mode-split operation can again be chosen to preserve stability and bandwidth, without large impact of the electronic noise. Further, the absence of nested parallel plates within the sense frame is beneficial to lower the damping of the sense mode, and the associated thermomechanical noise, and it is also beneficial for miniaturization.

Section 2 discusses the process flow, used to combine MEMS and NEMS structural elements, which also exploits eutectic bonding for reduction of dead-areas. It also introduces typical sensing configurations based on NEMS gauges, and presents the design of in-plane and out-of-plane gyroscopes exploiting this elements. Guidelines and trade-offs are discussed in light of the gain-factor and of thermo-mechanical noise contributions. Preliminary electromechanical test of the structures in terms of mode-split and quality factor is given in Section 3. In light of these results, Section 4 discusses the driving and sensing circuit design, analyses the electronic noise, and estimates the achievable white noise density.

Section 5 presents the experimental tests, showing high sensitivity and ultra-high-linearity ( 5 ppm) over a $1000 \mathrm{dps}$ full-scale. Within an area of less than $0.35 \mathrm{~mm}^{2}$ per axis, white noise densities in the mdps $/ \sqrt{ } \mathrm{Hz}$ range are obtained both on $\mathrm{Z}$ and $\mathrm{Y}$-axis devices, in agreement with theoretical predictions. A long-term stability in uncontrolled laboratory environment of about $2 \mathrm{dph}$ at observation times of $200 \mathrm{~s}$ is obtained. 

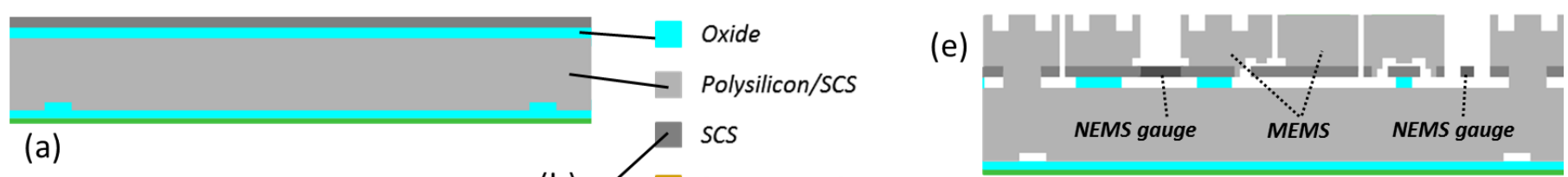

(a)

\section{SCS}

(b)

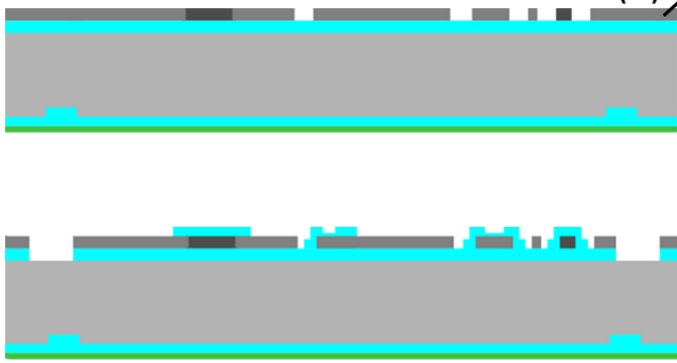

(c)

(d)
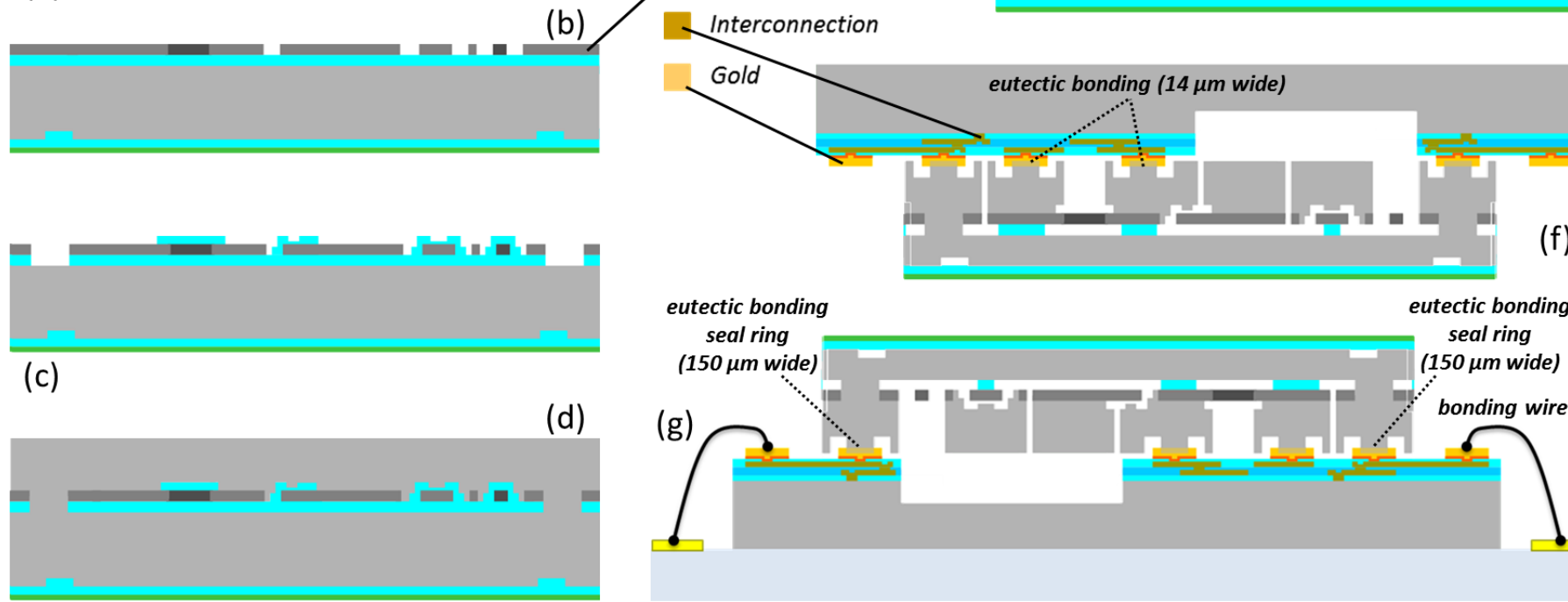

(f)

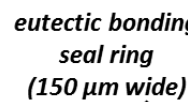

eutectic bonding

(g) seal ring (150 $\mu \mathrm{m}$ wide)

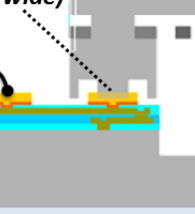

$\because$ bonding wire

Fig. 1. Schematic representation of the process flow of the M\&NEMS technology used for the devices in this work. Starting from an SOI wafer (a) and using common steps like DRIE, DUV and HF attacks (b-f), the process allows designing both micrometric (15 $\mu \mathrm{m}$ thick) and nanometric ( $250 \mathrm{~nm}$ thick) layers.

\section{PROCESS FLOW, SENSING PRINCIPLE AND DEVICE DESIGN}

The devices studied in this work exploit a combination of micrometric and sub-micrometric elements, together with $\mathrm{Au}$ Si eutectic bonding between the MEMS wafer, where the devices are designed, and a CAP wafer, where metal interconnections are routed.

\section{A. Process flow}

The main steps of the so-called M\&NEMS process flow used to fabricate the devices of this work are sketched in Fig. 1 [21]. Starting from a Silicon-on-Insulator wafer with a crystalline Silicon thickness of $250 \mathrm{~nm}$ (a), the first phase consists in the definition of the heavily doped NEMS patterns. The NEMS thin layer is removed (b) around those regions that define either the gauges or vertical electrodes (e.g. for selftest, or quadrature compensation). Inside these regions, the NEMS layer is kept separated, through Silicon oxide deposition and selective etching (c), from the epitaxial layer (d), which can reach typical values of surface micromachining processes, e.g. $15 \mu \mathrm{m}$ in this work. A two-step deep reactive ion etching (DRIE) defines the MEMS part as well as the lateral trenches of eutectic bonding contacts. The DRIE does not etch the nano-gauges thanks to the oxide protection. Oxide is then removed, through a hydrofluoric acid (HF) attack (e).

The CAP wafer, separately processed with two layers of metal interconnections, is now bonded to the MEMS wafer (f). Bonding occurs between Au on the CAP and Si on the MEMS wafer. The overall force during the eutectic bonding is about $30 \mathrm{kN}$, falling mostly on rectangular seal-rings with a width of $150 \mu \mathrm{m}$ and an overall area of $1.2 \mathrm{~mm}^{2}$, for each of the $\sim 2000$ modules on the wafer. This leads to about $20 \Omega$ parasitic resistance for an effective eutectic bonding contact area of (14 $\mu \mathrm{m})^{2}$ at each electrode. To avoid alignment issues between the two wafers, each electrode bonding region takes up $(68 \mu \mathrm{m})^{2}$. This occupation may be reduced in future designs to minimize dead areas. The CAP wafer features suitable cavities for getter deposition and minimization of the final pressure after bonding. The pressured device can be at this point tilted and wire bonded to the carrier or to a plastic package $(\mathrm{g})$.

\section{B. Lever system configuration for sensing}

The described process allows to simultaneously obtain monocrystalline Silicon, heavily doped beams with a cross section $A_{g}=(250 \mathrm{~nm})^{2}$ and a length $L_{g}$ of a few $\mu \mathrm{m}$, and 15 $\mu \mathrm{m}$-thick inertial masses (Fig. 2a). The beams can be exploited as piezoresistive sensing elements [20]. When subject to an axial stress $\sigma$ that causes a beam elongation or compression $\Delta L_{g}$, the beam resistance $R$ vary by a quantity $\Delta R$, according to its gauge factor $G F$ and to the Young's modulus $E$.

$$
\frac{\Delta R}{R}=G F \cdot \frac{\sigma}{E}=G F \cdot \frac{\Delta L_{g}}{L_{g}}
$$

The direct application of an axial force on the gauges proves challenging from a design point of view, as a 5 - $\mu \mathrm{m}$-long NEMS gauge has an axial stiffness of thousand $\mathrm{N} / \mathrm{m}$, which
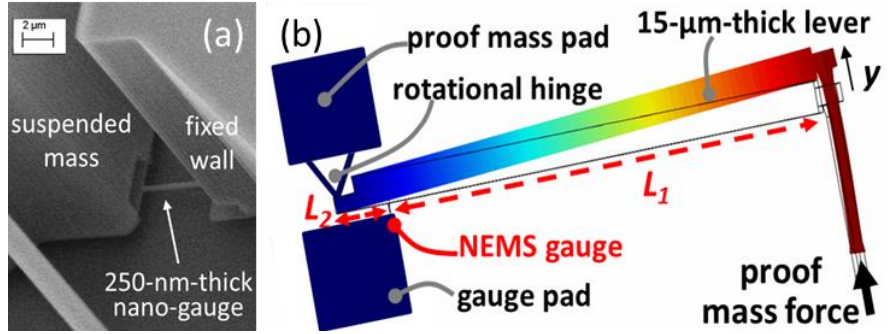

Fig. 2. (a) SEM picture of a NEMS gauge coupled to a suspended part on one side and to a fixed anchor point on the other side [19]. (b) Sensing scheme of a NEMS gauge coupled to a lever system (top view). L1 and L2 are the NEMS gauge distances to the lever tip and rotational hinge. 
makes it difficult to cope with typical resonance frequencies in the order of few tens $\mathrm{kHz}$ [22]. When using NEMS gauges as readout elements, it is therefore necessary to exploit a lever system to transfer the inertial force to one gauge end, while the other end is anchored. In the following, gyroscopes exploiting NEMS gauges for the sense mode will be described. Gyroscopes exploiting the NEMS gauges also for the drive-mode pick-off are currently under investigation.

Considering the situation sketched in Fig. 2b, we can now re-write Eq. (1) by considering the lever amplification factor $1 / \Gamma=\left(L_{1}+L_{2}\right) / L_{2}$ between the lever tip, which displaces by a quantity $y$, and the gauge elongation.

$$
\frac{\Delta R}{R}=G F \cdot \frac{\Delta L_{g}}{L_{g}}=G F \cdot \frac{\Gamma \cdot y}{L_{g}} \sim G F \cdot \frac{L_{2}}{L_{1}} \cdot \frac{y}{L_{g}}
$$

Note that in principle this coefficient is maximized by setting a large gauge distance $L_{2}$ from the hinge. In practice, there are other aspects to be considered for the optimization:

target sense-mode frequency: when changing the gauge position $L_{2}$, the stiffness of the hinge-gauge-lever system in Fig. $2 \mathrm{~b}$ changes. The stiffness of other springs involved in the gyroscope sense mode (e.g. drive-sense decoupling springs) should be changed accordingly, to hold the target frequency. A major limit $\left(L_{2, \max }<23 \mu \mathrm{m}\right)$ arises when the required width of the decoupling springs drops below minimum design values (1.7 $\mu \mathrm{m}$ for the used process);

- repeatability: the larger $L_{2}$, the larger the stress on the NEMS gauge (when keeping the sense-mode frequency constant as described above). However, too large contributions of the NEMS gauge to the overall sense-mode stiffness imply a different sense mode tolerance to etching nonuniformities with respect to the MEMS drive mode [19]. Targeting for Eq. (2) a nominal $\pm 5 \%$ repeatability from part to part (1-sigma value), $L_{2}$ cannot be extended beyond $L_{2, \max }<18 \mu \mathrm{m}$;

masks misalignments: the relative effect of misalignments between the NEMS and MEMS masks is larger for gauges closer to the hinge. From this point of view a value $L_{2}>10 \mu \mathrm{m}$ matches the repeatability given above for possible masks misalignments up to $0.5 \mu \mathrm{m}$.

Taking into account all these considerations, the value $L_{2}=$ $17 \mu \mathrm{m}$ was chosen as a compromise between the points above.

\section{Z-axis device design}

The sensing architecture with a lever system needs to cope with the gyroscope design. The first consideration that drives the architecture is that one should avoid to transfer the drivemode large motion to the nano-gauges. Therefore a doubly decoupled architecture, with an anchored drive frame, a Coriolis (or decoupling) frame, and the sense frame formed by a lever system similar to the description above is chosen.

TABLE I

FEM RESULTS FOR ACCELERATION REJECTION OF DIFFERENT STRUCTURES

\begin{tabular}{|c|c|c|c|c|c|c|}
\hline \multirow{2}{*}{$\begin{array}{c}\text { Acceleration } \\
100 \mathrm{~g} \\
(X \text { direction })\end{array}$} & \multicolumn{2}{|c|}{$\begin{array}{l}\text { Type (a) - stress } \\
\text { on the gauges }\end{array}$} & \multicolumn{2}{|c|}{$\begin{array}{l}\text { Type (b) - stress } \\
\text { on the gauges }\end{array}$} & \multicolumn{2}{|c|}{$\begin{array}{c}\text { Type (c) - stress } \\
\text { on the gauges }\end{array}$} \\
\hline & $75 \mathrm{kPa}$ & $D I F$ & $27 \mathrm{MPa}$ & $D I F$ & $60 \mathrm{kPa}$ & $C M$ \\
\hline $\begin{array}{c}100 \mathrm{~g} \\
\text { (Y direction) }\end{array}$ & $3 M P a$ & $C M$ & $20 \mathrm{MPa}$ & $C M$ & $150 \mathrm{MPa}$ & $C M$ \\
\hline $\begin{array}{c}100 \mathrm{~g} \\
\text { (Z direction) }\end{array}$ & $490 \mathrm{kPa}$ & $C M$ & $2.8 \mathrm{MPa}$ & $C M$ & $450 \mathrm{kPa}$ & $C M$ \\
\hline
\end{tabular}

$* \mathrm{DIF}=$ differential mode. $\quad * * \mathrm{CM}=$ common mode.
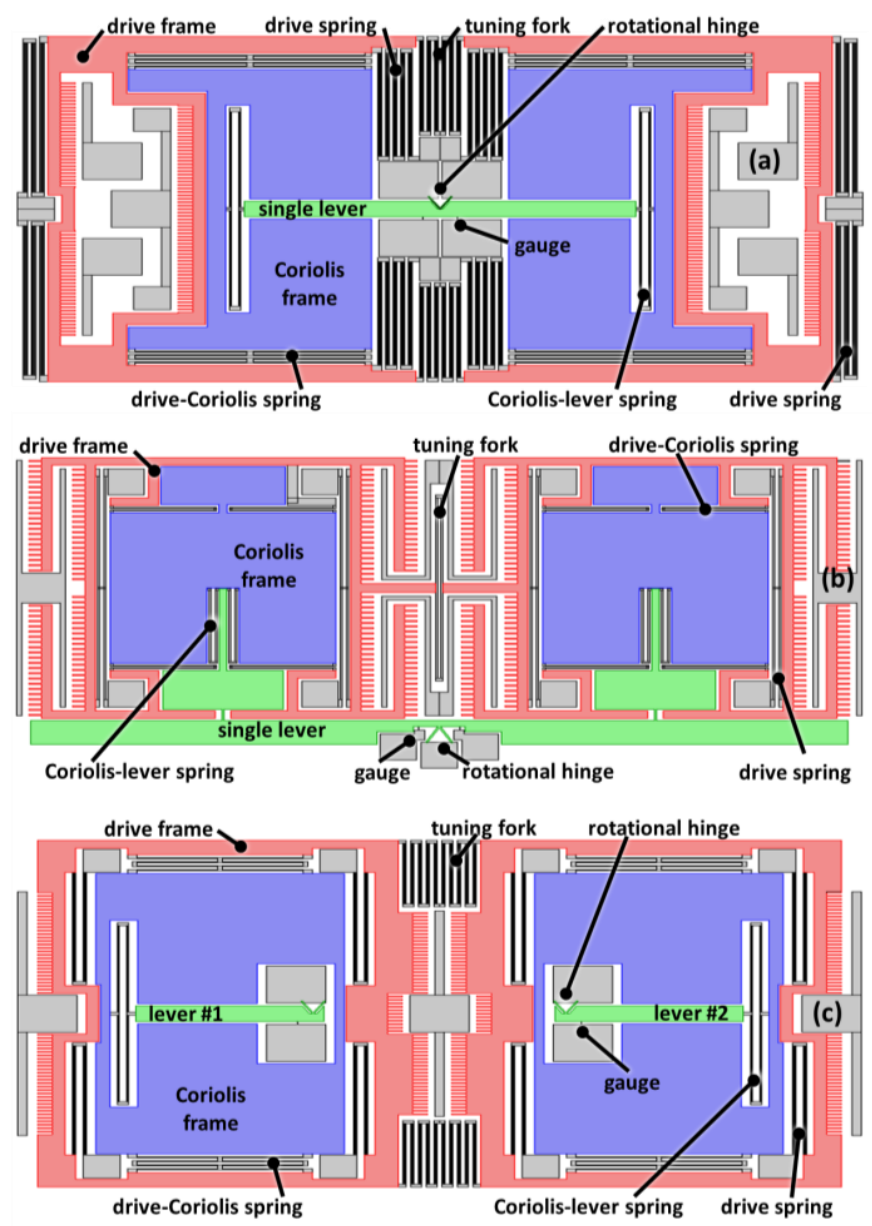

Fig. 3. Different architectures for gyroscopes based on NEMS gauges: (a) single lever along a symmetry axis; (b) single-lever external to the proof mass; (c) dual-lever along a symmetry axis. Comb-finger stators are not shown for sake of clarity

Three device configurations including a differential lever system and complying with a doubly-decoupled architecture were investigated and compared. Top views of the different architectures are given in Fig. 3 (the drive mode occurs along the horizontal direction). Option (a) includes a single lever positioned along one symmetry axis of the device. Five types of springs (drive, tuning-fork, drive-Coriolis decoupling, Coriolis-lever decoupling and rotational hinge) are highlighted in the figure, as well as the gauge position. Option (b) includes a single lever externally placed at one device side, resembling first configurations proposed for this kind of gyroscope [23, 24]. Option (c) includes separate levers for the sense frames.

For a given available area, no significant differences in obtainable sensitivity are found. On the other side, option (a) promises significantly better immunity to accelerations (and vibrations), both along the $\mathrm{X}$-axis if compared to solution (b), and along the $\mathrm{Y}$-axis if compared to both solutions (b) and (c). Table 1 reports the results of finite element simulations (FEM) for $100 \mathrm{~g}$ (gravity units) of acceleration in the three directions for the different topologies (large and/or differential stresses, highlighted in red, are the most critical ones). Option (a) also maximizes symmetry and compactness, and in the end it was chosen as the preferred design option.

Irrespective of the chosen architecture, note the absence of parallel plates within the sense frame. This implies a large 
sensing mass and the reduction of squeeze-film damping, often related to parallel-plate sensing. Both facts are positive in terms of achievable thermo-mechanical noise. A large mass also allows, for the same resonance frequency, the use of wider springs, which is positive for immunity to process overor under-etch, and therefore in terms of device repeatability.

The drive fingers overlap is dimensioned to guarantee a displacement $x=4 \mu \mathrm{m}$. The drive fingers gap is $1.1 \mu \mathrm{m}$. The frequencies are designed at about $20 \mathrm{kHz}$, with an intended mode split $\Delta f=\Delta \omega / 2 \pi=600 \mathrm{~Hz}$ (the target bandwidth for consumer applications is in the order of 200-300 Hz: as
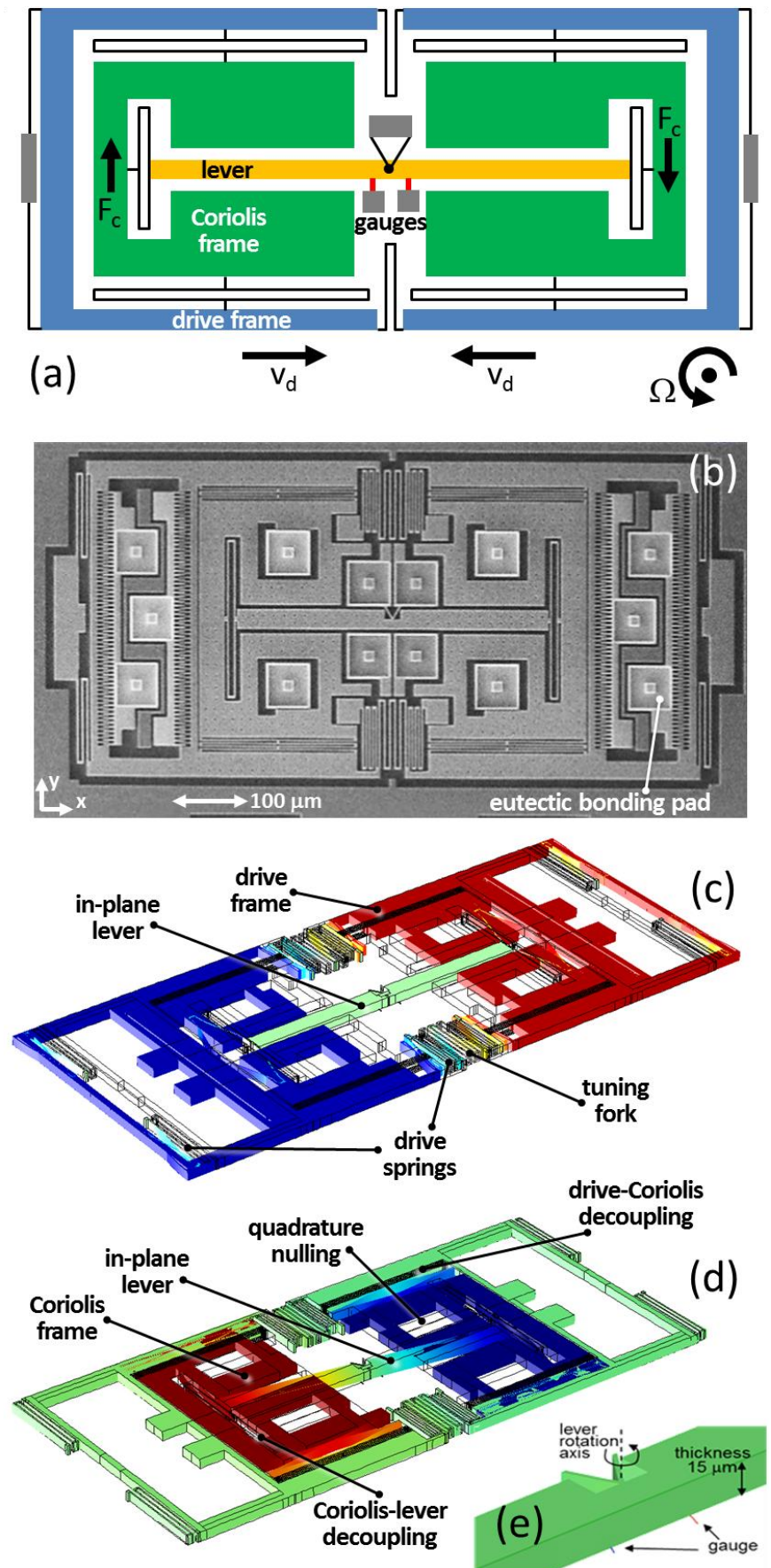

Fig. 4. (a) conceptual sketch (drive fingers not shown) and (b) SEM top view of the Z-axis device, with clearly visible pads for eutectic bonding (note the absence of interconnections on the MEMS wafer); (c) FEM of the antiphase tuning-fork drive mode; (d) FEM of the levered sense mode; (e) detail of the NEMS gauges positioned close to the in-plane rotational hinge. mentioned in the Introduction, such a target bandwidth cannot be obtained with a mode-matched device, unless force feedback is used). Fig. $4 \mathrm{a}$ and $4 \mathrm{~b}$ report a sketch and a scanning electron microscope (SEM) picture of the $\mathrm{Z}$-axis device. The overall area is $(885 \times 394) \mu \mathrm{m}^{2}$. Fig. $4 \mathrm{c}$ and $4 \mathrm{~d}$ report the drive and sense modes FEM, nominally occurring at $18.35 \mathrm{kHz}$ and $18.95 \mathrm{kHz}$ respectively, and the inset in Fig. $4 \mathrm{e}$ is a detail of the NEMS gauges positioned along the lever system close to the rotational hinge. Assuming a controlled drive motion amplitude (as described in the following of this work), the lever tip displacement $y$ as a function of the angular rate variation $\Delta \Omega$ can be written as [2]:

$$
\frac{y}{\Delta \Omega}=\frac{x}{\Delta \omega}
$$

Combining Eq. (2) and (3), one can obtain the gain-factor, i.e. the single-ended resistance variation per unit angular rate change.

$$
\frac{\Delta R}{\Delta \Omega}=G F \cdot \frac{\Gamma}{L_{g}} \cdot R \cdot \frac{x}{\Delta \omega}=G F \cdot \frac{\Gamma}{A_{g}} \cdot \rho \cdot \frac{x}{\Delta \omega} .
$$

For a nominal resistance value of $2 \mathrm{k} \Omega$ (corresponding to a gauge length of $5 \mu \mathrm{m}$ and a resistivity $\left.\rho=2.7 \cdot 10^{-5} \Omega \cdot \mathrm{m}\right)$, a $\Gamma$ factor $L_{2} /\left(L_{1}+L_{2}\right)=(17 \mu \mathrm{m}) /(228 \mu \mathrm{m}+17 \mu \mathrm{m})=0.069$, and a gauge factor in the order of 50 , the expected sensitivity turns out to be $1.48 \Omega /(\mathrm{rad} / \mathrm{s})=0.026 \Omega /(\mathrm{dps})$ for each NEMS. Note that the sensitivity goes with one over the cross section, which was chosen as the minimum one.

The rate noise density $S_{\Omega, t m}$, considering only the mechanical noise of the sense frame $S_{y}$, and assuming a constant transfer function $Q_{e f f} / k_{s}$ between sense frame force and displacement ( $Q_{\text {eff }}$ being the gain at a distance $\Delta f$ from resonance, and $k_{s}$ being the sense frame stiffness), turns out to be [2]:

$$
S_{\Omega, \mathrm{tm}}=\frac{\mathrm{S}_{\mathrm{y}}}{\frac{\mathrm{y}}{\Delta \Omega}}=\frac{\sqrt{4 \cdot \mathrm{k}_{\mathrm{B}} \cdot \mathrm{T} \cdot \mathrm{b}_{\mathrm{S}} \cdot\left(\frac{\mathrm{Q}_{\mathrm{eff}}}{\mathrm{k}_{\mathrm{S}}}\right)^{2}}}{\frac{\mathrm{y}}{\Delta \Omega}}=\frac{1}{\mathrm{x} \cdot \omega_{s}} \sqrt{\frac{\mathrm{k}_{\mathrm{B}} \cdot \mathrm{T} \cdot \mathrm{b}_{\mathrm{S}}}{\mathrm{m}_{\mathrm{S}}{ }^{2}}}
$$

In the equation above $k_{b}$ and $T$ are the Boltzmann constant and the absolute temperature, and $m_{s}$ and $b_{s}$ are the sense mode effective mass and damping coefficient respectively. For the design values $m_{s}=3.9 \cdot 10^{-9} \mathrm{~kg}, f_{s}=18.95 \mathrm{kHz}$ and $b_{s}=1.5 \cdot 10^{-7}$ $\mathrm{kg} / \mathrm{s}$, the predicted rate noise density caused by thermomechanical effects turns out to be $750 \mu \mathrm{dps} / \sqrt{\mathrm{Hz}}$. The value of the damping coefficient, here given for a nominal pressure of 1 mbar, is dominated by squeeze-film effect generated by the springs folds and by quadrature nulling electrodes designed within the sense frame (see again Fig. 4b).

\section{D. $\quad Y$-axis device design}

The considerations drawn for $\mathrm{Z}$-axis gyroscopes were extended to the design of in-plane gyroscopes. Fig. 5a and 5b are a schematic sketch and a SEM picture of the Y-axis device, showing the doubly decoupled architecture with the anchored drive frame, the Coriolis (or decoupling) frame, and the sense levered system. The overall area is $(788 \times 401) \mu \mathrm{m}^{2}$. Fig. $5 \mathrm{c}$ and $5 \mathrm{~d}$ report the drive and sense modes, nominally occurring at $18.43 \mathrm{kHz}$ and $18.83 \mathrm{kHz}$ respectively, the latter showing the out-of-plane rotation of the lever. This rotation causes differential stresses on the gauges, located at the basis of the MEMS part, as sketched in the inset given by Fig. 5e.

In particular, the use of a single lever system, positioned along one symmetry axis was obtained by placing the gauges 
along the torsional beams of an out-of-plane lever. Note that in this case, for a given rotation angle $\alpha$ of the Coriolis frame, the gauge displacement $\Delta L_{g}$ is determined a priori by the fact that the gauge is attached to the base of the micrometric structure and by the fact that the epitaxy height $h$ is a process rather than a design parameter. Defining $z$ as the vertical displacement of the lever tip, with a lever length $L_{L}$, Eq. (2) and (4) becomes in this situation:

$$
\frac{\Delta R}{R}=G F \cdot \frac{\Delta L_{g}}{L_{g}}=G F \cdot \frac{\frac{\mathrm{h}}{2} \cdot \alpha}{L_{g}}=G F \cdot \frac{\frac{\mathrm{h}}{2} \cdot \frac{z}{L_{L}}}{L_{g}} .
$$
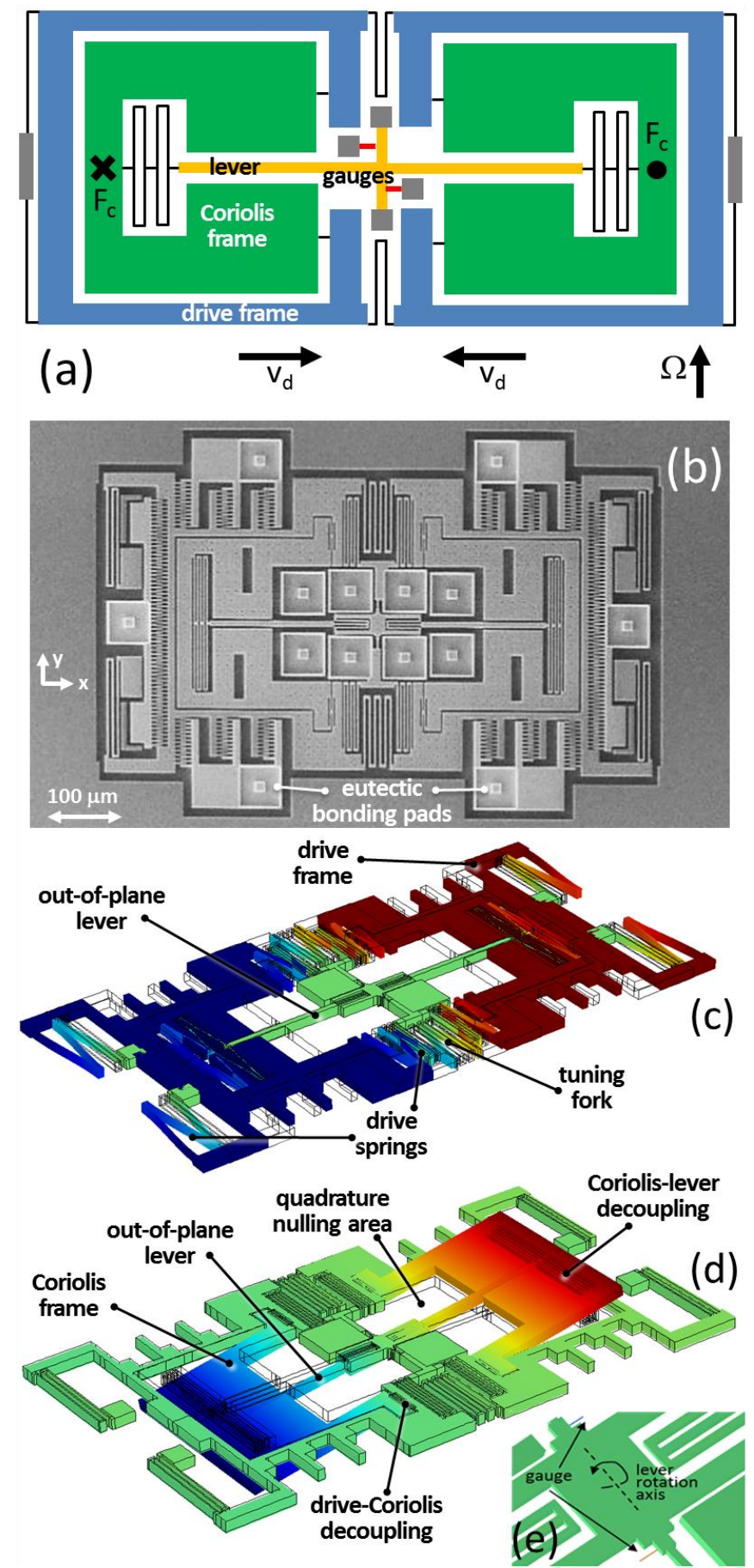

Fig. 5. (a) conceptual sketch (drive fingers not shown) and (b) SEM top view of the Y-axis device, with clearly visible pads for eutectic bonding (note the absence of interconnections on the MEMS wafer); (c) FEM of the antiphase tuning-fork drive mode; (d) FEM of the levered sense mode; (e) detail of the NEMS gauges positioned close to the out-of-plane rotational hinge.

$$
\frac{\Delta R}{\Delta \Omega}=G F \cdot \frac{\mathrm{h}}{2 \cdot L_{L} \cdot L_{g}} \cdot R \cdot \frac{x}{\Delta \omega} .
$$

A short lever length $L_{L}$ apparently improves the sensitivity. Yet, it also increases the sense mode stiffness. As it should cope with the target frequency, in the end it trades off with the minimum allowed width of the other springs taking part to the sense mode (drive-Coriolis decoupling in Fig. 5c). The value of $\left(h / 2 L_{L}\right)$ is $2.3 x$ lower than $\Gamma$ calculated for Z-axis devices.

A mode split value of $400 \mathrm{~Hz}$ and a drive motion amplitude of $6 \mu \mathrm{m}$ are thus needed, for the used lever length $(245 \mu \mathrm{m})$, to match the gain-factor of Z-axis devices. As an alternative, the device can be designed with the same mode-split and drive amplitude, and the lower lever factor can be compensated in operation, acting on the gauge bias current.

The rate noise density is affected by the $1.5 \mu \mathrm{m}$ vertical distance between the proof mass and the quadrature compensation electrodes: a larger compensation area with respect to Z-axis devices was designed, as in-plane gyroscopes are usually more affected by skew-angle issues and associated quadrature [25]. As a consequence the predicted damping coefficient $[2,26]$ is about 10 times larger than in Z-axis devices. According to Eq. (5), the rate noise density caused by thermo-mechanical noise turns out to be $S_{\Omega, t m}=1.7 \mathrm{mdps} / \sqrt{ } \mathrm{Hz}$.

Table II summarizes the parameters of the designed devices.

\section{ELECTROMECHANICAL CHARACTERIZATION}

Before designing the drive oscillator and the sense interface, a characterization was performed to verify the effective electromechanical parameters against expected values. In particular, the eutectic bonding, still in a process optimization phase, required to check the values of the obtainable pressure and quality factors. These tests were done using a modified $M C P-G$ from ITmems [27], enabling both capacitive and piezoresistive readout of the MEMS modes. In the tested devices, quadrature electrodes were used as self-test for the sense mode actuation. Specimens were tested through a probe station, at wafer-level in a clean room environment.

To later identify the effective pressure in each die, reference measurements inside a vacuum probe station were first performed on $4 \mathrm{Z}$-axis devices of a bare wafer, giving average drive-mode $Q$ values of 30790 at $0.1 \mathrm{mbar}, 4080$ at $1 \mathrm{mbar}$ and 520 at 10 mbar. The mode was characterized using the ring-down response approach, as described e.g. in [19]. The measurement results confirm that at pressures in the order of 1 mbar, expected for the final packaging, the damping range is linear, which allows to easily estimate the effective pressure.

Similar measurements were then repeated on capped wafers after the eutectic bonding process. Fig. 6a summarizes the

TABLE II

\begin{tabular}{|c|c|c|c|}
\hline \multicolumn{2}{|l|}{ Z-axis gyroscope } & \multicolumn{2}{|l|}{ Y-axis gyroscope } \\
\hline Overall length $[\mu \mathrm{m}]$ & 885 & Overall length $[\mu \mathrm{m}]$ & 788 \\
\hline Overall width $[\mu \mathrm{m}]$ & 394 & Overall width $[\mu \mathrm{m}]$ & 401 \\
\hline Drive fingers gap $[\mu \mathrm{m}]$ & 1.1 & Drive fingers gap $[\mu \mathrm{m}]$ & 1.1 \\
\hline Drive fingers \# & 118 & Drive fingers \# & 126 \\
\hline Nominal mode split [Hz] & 600 & Nominal mode split [Hz] & 400 \\
\hline Target drive displacement $[\mu \mathrm{m}]$ & 4 & Target drive displacement [ $\mu \mathrm{m}]$ & 6 \\
\hline Lever tip motion [pm/dps] & 19 & Lever tip motion [pm/dps] & 39 \\
\hline Nominal sensitivity $[\Omega / d p s]$ & 0.026 & Nominal sensitivity $[\Omega / d p s]$ & 0.026 \\
\hline
\end{tabular}

GEOMETRICAL DIMENSIONS AND EXPECTED SENSITIVITY OF THE DEVICES 

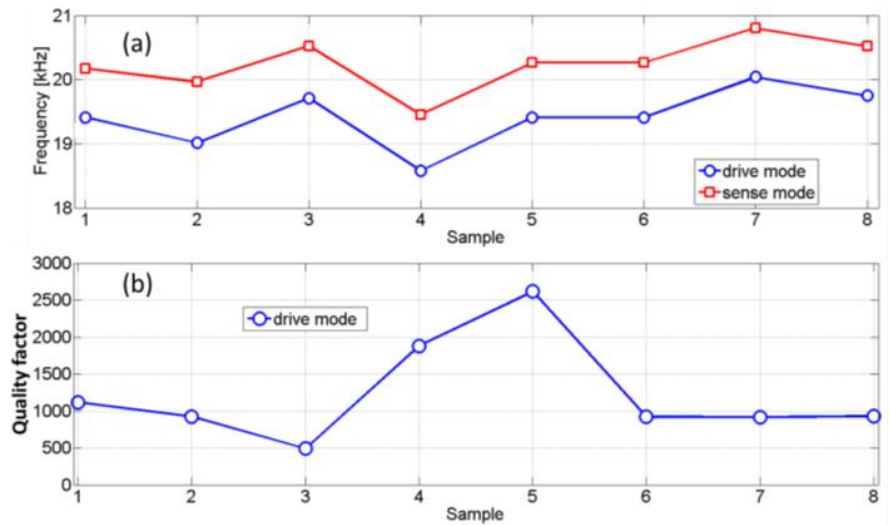

Fig. 6. (a) measured drive and sense mode frequencies and (b) corresponding drive-mode quality factors on $\mathrm{Z}$-axis devices. The latter measurements were used, through a comparative analysis in a vacuum probe station on bare wafers, to later identify the pressure of packaged samples.

results obtained on $8 \mathrm{Z}$-axis samples (all from the central portion of the same wafer) in terms of drive and sense frequencies. The corresponding average mode-split is $800 \mathrm{~Hz}$, slightly larger than predicted.

Fig. 6b reports the corresponding drive mode quality factors. The average value is 1224 , with a relatively large dispersion. Reasons of such spread are under investigation. The estimated average pressure of the packaged dies is therefore $3.3 \mathrm{mbar}$.

The wafer-level Q-factor yield and variability for 8 Y-axis devices was the same as for Z-axis devices, as the two gyroscope types are located in the same module. $15 \%$ lower values of the average drive-mode quality factor are however obtained for $\mathrm{Y}$-axis gyroscopes. As these devices have a similar drive topology in terms of driven mass, stiffness, and comb fingers (see Table II), the decrease in quality factor is ascribed to slide-film damping between the proof mass and vertical electrodes (which are not present in Z-axis devices). Average values of the mode split were found to be in the order of $1 \mathrm{kHz}$. This partial difference with respect to FEM predictions is under investigation for an improved design.

\section{ELECTRONIC ARCHITECTURE AND DESIGN}

The driving and readout electronics is formed by 5 main building blocks: (i) a primary oscillator built around the drive resonator, (ii) a secondary loop for drive motion amplitude control, (iii) a feed-through capacitance compensation circuit, (iv) a Wheatstone bridge sensing interface with further amplification, and ( $v$ ) a lock-in based demodulation. The electronics is implemented using board-level circuits and is made compatible with mounting on the rate table used for the measurements of Section V.

The overall schematic of the circuits is presented in Fig. 7a.

\section{A. Drive circuit oscillator}

The first three blocks implement the drive circuit. The gyroscope proof mass is kept at the ground potential and is driven via the comb stators with an $\mathrm{AC}$ square wave of a few hundred $\mathrm{mV}_{\text {rms }}$ superimposed to a DC voltage of $15 \mathrm{~V}$. Drivemode pick-off is performed via a second set of comb fingers. The output motional current $i_{m}$ is sensed through a low-noise transimpedance (TIA) stage, further amplified through two gain stages (G1 and $G 2$ ) that make it saturate to the $\pm 5 \mathrm{~V}$ supply. This saturation represents the nonlinearity that sets the loop-gain to 1 after the initial start-up phase, to satisfy the Barkhausen condition [28]. The feedback capacitances of the TIA and of the $G 1$ stage are used to avoid the open-loop-gain pass unity at frequencies other than the resonance [29].

The signal at the output of $G 2$, now in the form of a square wave, is conditioned through a resistive voltage divider and a variable gain amplifier (VGA). The VGA output is summed to the DC voltage through the stage $G 3$, whose output finally drives the MEMS.

To compensate for the observed variations in the quality factor and/or in the resonance frequency from part to part, a secondary loop (control loop) integrates the TIA output in order to recover a signal proportional to the motion amplitude. As an alternative, a charge amplifier could be directly used instead of the TIA as a front-end, but would lead to worse noise performance of the drive loop due to the current noise of
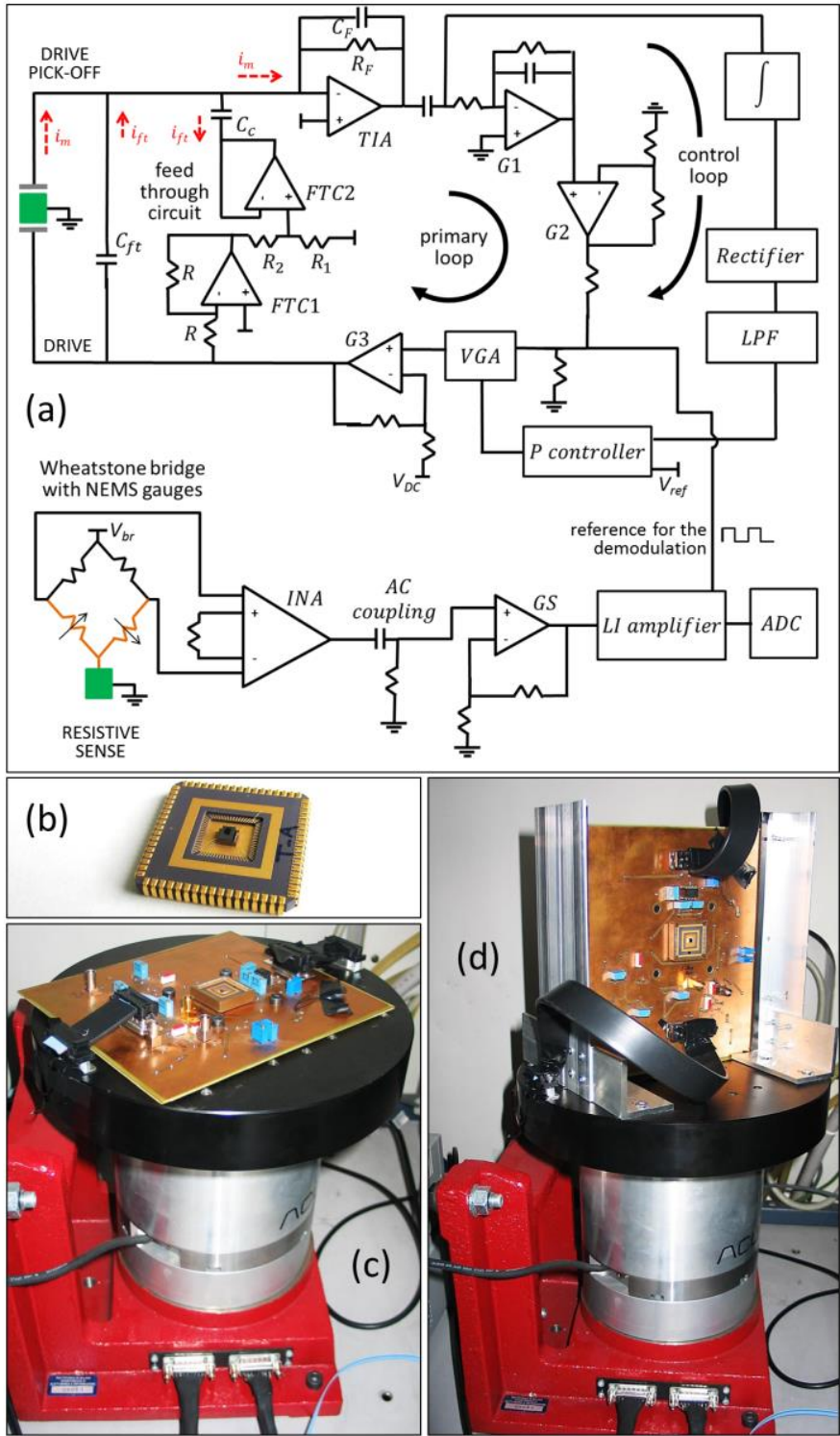

Fig. 7. (a) schematic view of the implemented electronics for the operation of the gyroscopes. Note the presence of a primary loop for the drive oscillator, a control loop to set the drive amplitude, and a feedthrough compensation circuit. The sensing interface is based on a Wheatstone bridge. Picture (b) reports the device assembled in a ceramic package, which is then mounted on the rate table for either Z-axis (c) or Y-axis (d) rate testing [1]. 
the chosen operational amplifier. After integration, the signal is rectified, low-pass filtered, and compared to a reference voltage $V_{\text {ref }}$. At any time, the error between the actual motion and the reference is processed by the proportional controller to drive the $V G A$, and in turn to adjust the AC voltage applied to the MEMS, in order to stabilize the motion amplitude.

The operational amplifiers FTC1 and FTC2 are used to implement a compensation of the feedthrough capacitance. With the assumption that the feedthrough $C_{f t}$ is identical in all the devices of the same type, the compensation is based on an initial trimming of the voltage divider $\left(R_{l}, R_{2}\right)$, so that the compensation capacitance $C_{c}$ recalls a current equal and opposite to $i_{f t}$ generated by the feedthrough capacitance $C_{f t}$. The implementation of this sub-circuit turns out to be helpful in case the obtained package pressure is higher than expected (as in this case), implying relatively low drive mode quality factors, and correspondingly high feed-through impact.

\section{B. Sensing interface}

The board-level sensing interface is based on a Wheatstone bridge: the gauges, attached to the proof mass on one side, share a common contact kept to ground. The other contact is connected to external resistors, trimmed to the measured gauge value $(\sim 2 \mathrm{k} \Omega)$ to complete the bridge.

As the NEMS gauges vary in opposite direction in presence of angular rates, the bridge output changes accordingly. An instrumentation amplifier (INA) with a 50x gain reads the bridge output, which is then adapted to the input dynamic of a lock-in amplifier by a non-inverting, selectable-gain stage $G S$.

The demodulated output of the lock-in amplifier (SRS830 from Stanford Research System) is digitized through a Measurement Computing MCC-1608GX acquisition board.

One contribution to the electronic noise generated in the sense chain is due to the Johnson noise $S_{R}$ of the bridge resistors, whose power spectral density sums up in the difference implemented by the INA. The input-referred noise, reported as angular rate white noise density, turns out to be:

$$
\mathrm{S}_{\Omega, \mathrm{Jh}}=\frac{\mathrm{S}_{\mathrm{R}}}{\frac{\Delta R}{\Delta \Omega}}=\frac{2 \cdot R}{V_{b r}} \cdot \sqrt{4 k_{B} T R} \cdot \frac{1}{\frac{\Delta R}{\Delta \Omega}} .
$$

For a $2 \mathrm{k} \Omega$ value of the resistors, and a bridge biasing voltage of $1.8 \mathrm{~V}$ (as discussed later, this voltage can be duty-cycled to save power consumption), the input-referred noise turns out to be $491 \mu \mathrm{dps} / \sqrt{ } \mathrm{Hz}$ for the gain-factor of $0.026 \Omega /(\mathrm{dps})$ derived in Section II.C and II.D .

A second contribution is given by the INA input-referred noise $S_{\Omega, I N A}$, which can be calculated from its voltage noise density $S_{V, I N A}$ as:

$$
\mathrm{S}_{\Omega, \mathrm{INA}}=\frac{2 \cdot R}{V_{b r}} \cdot \mathrm{S}_{\mathrm{V}, \mathrm{INA}} \cdot \frac{\sqrt{2}}{\frac{\Delta R}{\Delta \Omega}} .
$$

With the value of the used amplifier $(8 \mathrm{nV} / \sqrt{\mathrm{Hz}}$, INA129 from Texas Instruments) and the same biasing voltage and gainfactor as above, the predicted contribution turns out to be about $950 \mu \mathrm{dps} / \sqrt{ } \mathrm{Hz}$.

The overall electronic noise value is comparable (Z-axis) or lower (Y-axis) than the thermo-mechanical noise limits, confirming the high potential of NEMS gauge sensing.

The samples to be tested were mounted on ceramic carriers, placed inside a plastic socket soldered to the board. The board was accurately mounted on an Acutronic rate table for Z-axis and $\mathrm{Y}$-axis angular rate testing (Fig. $7 \mathrm{~b}$ to $7 \mathrm{~d}$ ). Measurements were controlled and automated through a Labview interface that allows to implement sensitivity, bandwidth and noise measurements. Quadrature can be compensated according to the Tatar scheme [30], but for the first measurements shown in this work devices with natively low quadrature were selected.

\section{EXPERIMENTAL RESULTS}

\section{A. Sensitivity and linearity}

As mentioned in Section III, some devices used for the tests have a mode split which differs from the design values. The tested Z-axis device has the correct $\Delta f \sim 600 \mathrm{~Hz}$, but the tested $\mathrm{Y}$-axis device has a $\Delta f \sim 1080 \mathrm{~Hz}$. The devices are tested with the same nominal drive mode amplitude $x=4 \mu \mathrm{m}$.

The phase reference for demodulation is set by the saturated output of the primary loop in Fig. 7. The zero-rate offset, all appearing in the quadrature channel, is of $24 \mathrm{dps}$ and $16 \mathrm{dps}$ respectively. The absence of any noticeable offsets in the Coriolis channel is ascribed (i) to low impact of comb-drive defects (see e.g. [23]) and (ii) to the fact the resistive readout is quite immune from possible capacitive couplings of any signals occurring in phase with the Coriolis signal.

Using the formulas of Eq. (4) and (7), and coupling them to the electronic gains of the INA, GS and lock-in stages $\left(G_{I N A}=\right.$ $50, G_{G S}=2$ and $G_{L I}=10 / \sqrt{ } 2$ ), the predicted system sensitivity, normalized to the bridge voltage $V_{b r}$,

$$
\frac{\Delta V_{\text {out }}}{V_{b r} \cdot \Delta \Omega}=\frac{1}{2 \cdot R} \cdot \frac{\Delta R}{\Delta \Omega} G_{I N A} G_{G S} G_{L I}
$$

turns out to be $4.6 \mathrm{mV} / \mathrm{V} / \mathrm{dps}$ for the $\mathrm{Z}$-axis device and 1.13 $\mathrm{mV} / \mathrm{V} / \mathrm{dps}$ for the $\mathrm{Y}$-axis.

The measured sensitivities turn out to be quite in agreement with the predictions. For the Z-axis device, Fig. 8a reports the output voltage as a function of the Z-axis input rate up to 1000 $\mathrm{dps}$, at $340 \mathrm{mV}$ bridge biasing voltage. The best fitting curve indicates $5.47 \mathrm{mV} / \mathrm{V} / \mathrm{dps}$. The difference (17\% larger) from the theoretical value may be ascribed to a different value of the lever factor $\Gamma$ and/or of the NEMS gauge cross-section.

Fig. 8b reports the output voltage of the $\mathrm{Y}$-axis device under
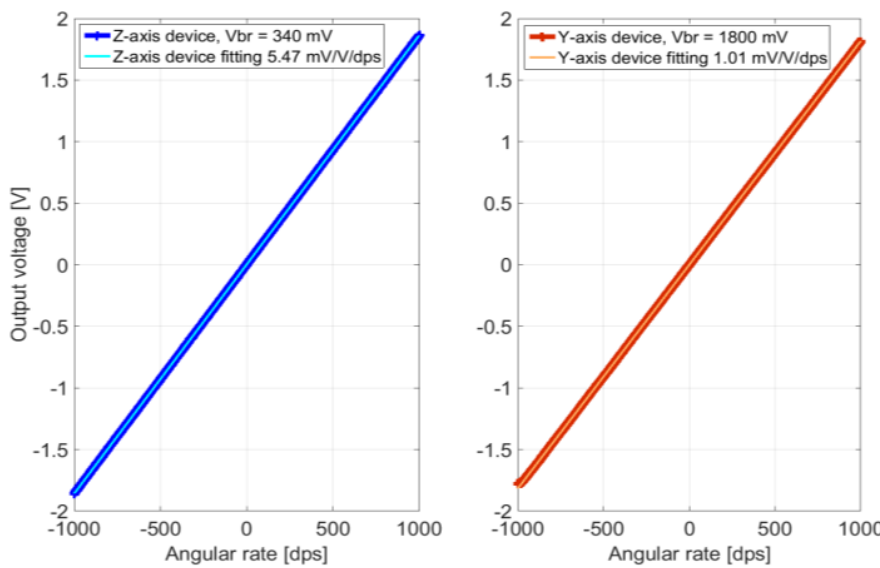

Fig. 8. Matching measured sensitivities for the Z-axis and the Y-axis device. Due to difference between design and measured parameters (in particular the mode-split) a different biasing voltage of the bridge needs to be used to compensate the $\sim 6$ times lower sensitivity of the $\mathrm{Y}$-axis device. 

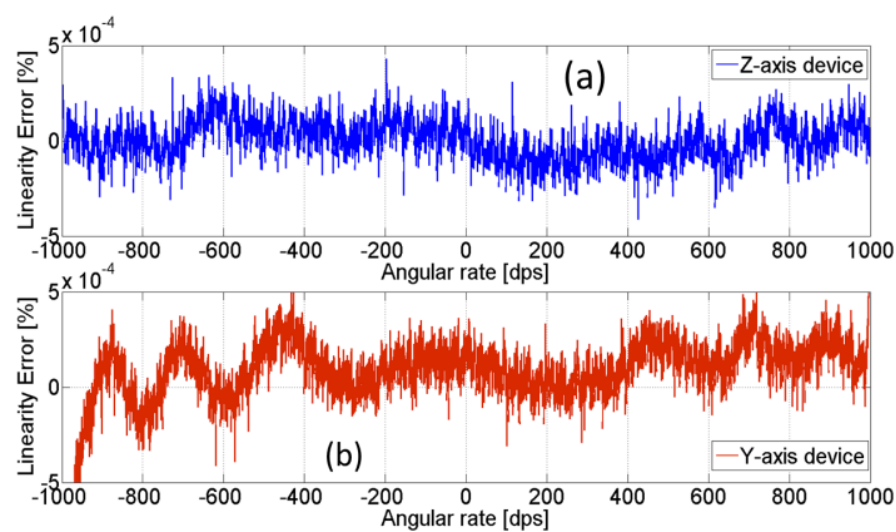

Fig. 9. Linearity error for the Z-axis device (a) and for the Y-axis device (b) as a percentage of the full-scale, here assumed as $1000 \mathrm{dps}$.

$\mathrm{Y}$-axis angular rates. Due to the large mode split, the bridge biasing voltage to match the same sensitivity as the Z-axis device turns out to be $1.8 \mathrm{~V}$, resulting in a best fitting curve of $1.01 \mathrm{mV} / \mathrm{V} / \mathrm{dps}$, to be compared to a theoretical prediction of $1.13 \mathrm{mV} / \mathrm{V} / \mathrm{dps}$.

Fig. 9 shows the derived linearity error (\% of the full-scale) showing unprecedented values within $5 \mathrm{ppm}$ for both the devices. To explain this result, consider that the relative linearity error for NEMS gauge readout was characterized in a previous work [31] to be within few \%, even in single-ended configuration, for stresses as large as $2 \mathrm{GPa}$. Considering the sensitivity in terms of stress $(41.6 \mathrm{kPa} / \mathrm{dps})$ derived from Eq. (4), the maximum loading value (at 1000 dps rate) turns out to be $41.6 \mathrm{MPa}$, which is almost two orders of magnitude lower than the mentioned nonlinearity limit.

Measured cross-axis rejections are larger than $55 \mathrm{~dB}$. The measured bandwidth $(300 \mathrm{~Hz})$ is limited by the rate table mechanical response.

\section{B. Noise measurements}

To verify the predicted noise performance, Allan variance measurements were taken in uncontrolled laboratory environment, in different conditions of bridge biasing. The results are summarized by Fig. 10.

The Z-axis device was first tested with a bridge voltage of $340 \mathrm{mV}$ (corresponding to the sensitivity shown in Fig. 8a). Eight Allan variance curves, lasting up to 60 minutes each, were obtained in three different measurement sessions, with a good repeatability in terms of measured angle random walk $(\mathrm{ARW}=4.2 \mathrm{mdps} / \sqrt{\mathrm{Hz}}$, dashed curves). Effects of bias stability begin to be visible at values around 1-4 dph, and 100$360 \mathrm{~s} \mathrm{(corresponding} \mathrm{to} \mathrm{the} \mathrm{full} \mathrm{acquisition} \mathrm{time} \mathrm{with} \mathrm{ten-point}$ average in the Allan formula). In the operating conditions, the overall ARW predicted by the noise formulas (5), (7) and (8), accounting for the effectively measured sensitivity, is 4.1 $\mathrm{mdps} / \sqrt{\mathrm{Hz}}$, quite close to the measured data.

A 3-fold increase in the bridge voltage determines a reduction in the white noise, but not by the same factor, indicating that the dominant noise contribution begins to be thermo-mechanical. The measured ARW value of $2 \mathrm{mdps} / \sqrt{\mathrm{Hz}}$ (see the three measurements obtained in this situation, reported in Fig. 10, circle markers) is in very good agreement with the predicted value of $1.8 \mathrm{mdps} / \sqrt{\mathrm{Hz}}$. Some instability is visible at rate values of $1.1 \mathrm{dph}$. Such a resolution satisfies even gyro-compassing application requirements [32], the earth rotation rate being $15 \mathrm{dph}$.

The Y-axis device was first tested in the conditions of Fig. $8 \mathrm{~b}\left(V_{b r}=1.8 \mathrm{~V}\right)$. Three measurements and their average are reported in Fig. 10. The obtained ARW level is in the order of $10 \mathrm{mdps} / \sqrt{\mathrm{Hz}}$, compared to a predicted value of 6.3 $\mathrm{mdps} / \sqrt{\mathrm{Hz}}$. The measured bias stability is about $20 \mathrm{dph}$ at $20 \mathrm{~s}$ observation interval.

Increasing the bridge voltage leads to a reduction in the ARW, roughly by the same factor, down to $8.2 \mathrm{mdps} / \sqrt{\mathrm{Hz}}$. The excess electronic noise is somewhat surprising as the used board is the same as for Z-axis devices. Vertical mounting of the board with long cables for Y-axis test (Fig. 7b) may induce a disturbance coupling, which is still under investigation.

\section{CONCLUSIONS}

The work presented novel structures of in-plane and out-ofplane gyroscopes based on piezoresistive NEMS readout. Though some differences were encountered with respect to FEM modes predictions, in particular for in-plane devices, the obtained results demonstrate ultra-high linearity at low white noise levels within large full-scale ranges. The area taken up by a 3-axis gyroscope of this type is lower than $(1.2 \mathrm{~mm})^{2}$.

For low-power applications, the current dissipated in the gauges may be reduced by adopting a modulated bridge voltage. Similar approaches are already exploited in other miniaturized sensors based on Wheatstone bridges, like anisotropic magneto-resistance (AMR) devices [33, 34].

Further characterization activities are dedicated (i) to reliability tests, and (ii) to noise and sensitivity analyses under environmental changes and vibrations.

In particular, concerning point (i) above, the mechanical properties of the NEMS layer were characterized thoroughly in [33]. From there estimated nominal strengths of about 6 $\mathrm{GPa}$, and according to FEM results shown in Table 1 to analyze effects of large accelerations, one finds a failure stationary acceleration value along the $\mathrm{y}$-axis of $0.2 \mathrm{Mg}$ (gravity units). This value largely exceeds typical industrial reliability shock tests of $10^{4} \mathrm{~g}$ for consumer applications [1215]. Further: such large accelerations typically occur as impulsive shocks, with sub-ms duration. So taking into account the quasi-stationary values of Table 1 is a

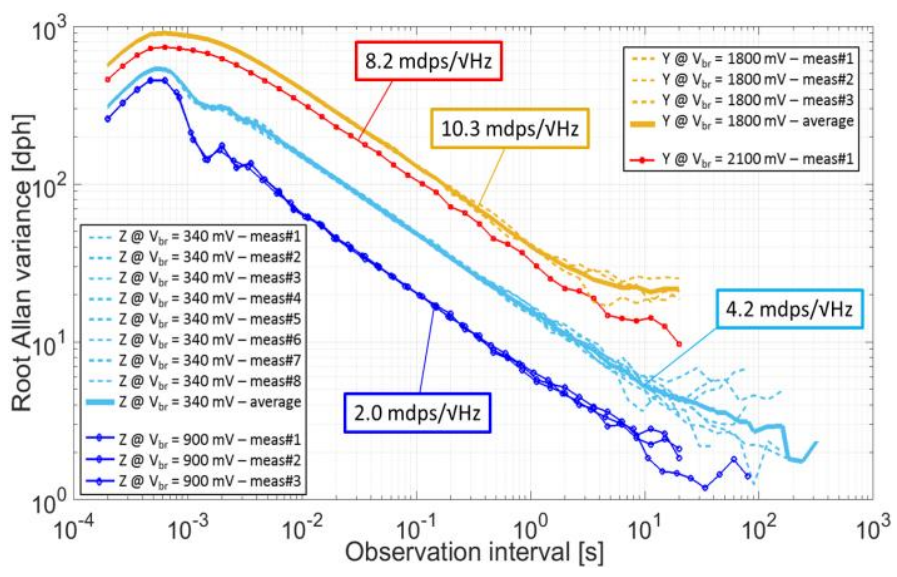

Fig. 10. Measured Allan variance curves in different conditions of the bridge biasing for the $\mathrm{Z}$-axis and $\mathrm{Y}$-axis devices of this work. 
conservative approach. Besides, much before the MEMS sense mass undergoes the full displacement induced by such shocks, and so much before all the stress is delivered to the NEMS gauge, the suspended MEMS part will be blocked by suitably designed mechanical stoppers absorbing the shock, like in common capacitive devices. A complete reliability characterization campaign under shocks and drop-tests is however still to be done.

Finally, concerning point (ii) above, since this is a resistive readout, particular care will be given to characterization of offset and sensitivity under temperature changes. Linear compensation schemes may be considered, as this is the approach already followed for other sensors relying on resistive bridges, e.g. AMR magnetometers. With respect to such devices however, gyroscopes operate as modulated sensors at $20 \mathrm{kHz}$ : therefore temperature changes affecting the bridge DC output should be bypassed by the demodulation, and therefore offset drifts should be inherently cancelled (indeed the good results shown in Fig. 10 exploited no temperature compensation scheme). To accurately test effects of temperature changes on gyroscope sensitivity, a mini climatic chamber, compatible with the rate-table setup shown in Fig. 7b, is under development.

\section{ACKNOWLEDGMENT}

The author acknowledge E. Brigo, G. Schierano and F. Maspero from Politecnico di Milano for helping with models, and A. Berthelot and P. Rey from CEA-Leti for helping with fabrication. The work is supported under European Union, FP7-ICT grant 288318 (NIRVANA project).

\section{REFERENCES}

[1] S. Dellea, F. Giacci, A. Longoni, P. Rey, A. Berthelot, G. Langfelder, Large Full Scale, Linearity and Cross-Axis Rejection in Low-Power 3Axis Gyroscopes Based on Nanoscale Piezoresistors, Proc. IEEE MEMS 2015, Estoril (Portugal), Jan 2015, pp. 37-40.

[2] V. Kempe, Gyroscopes, Chapter 8 in Inertial MEMS Principles and Practice, Cambridge University press, 2011.

[3] C. Acar, A. Shkel, MEMS Vibratory Gyroscopes, MEMS Reference Shelf, Springer, 2009.

[4] R. Antonello, R. Oboe, D. Pilastro, S. Viola, K. Ito, A. Cenedese, IMUbased image stabilization in a HSM-driven camera positioning unit, IEEE Int. Conf. on Mechatronics (ICM), 2013, Feb 2013, pp. 156,161.

[5] D. Senkal, E.J. Ng, V. Hong, Y. Yang, C.H. Ahn, T.W. Kenny, and A.M. Shkel, Parametric drive of a toroidal mems rate integrating Gyroscope demonstrating < 20 ppm scale factor stability, Proc. IEEE MEMS 2015, Estoril (Portugal), Jan 2015, pp. 29-32.

[6] A. Sharma, M. F. Zaman, F. Ayazi, A 104-dB Dynamic Range Transimpedance-Based CMOS ASIC for Tuning Fork Microgyroscopes, IEEE Journ. Solid-State Circ., vol. 42, n. 8, Aug 2007, pp. 1790-1802.

[7] C. Acar, A. R. Schofield, A. A. Trusov, L. E. Costlow, A. M. Shkel, Environmentally Robust MEMS Vibratory Gyroscopes for Automotive Applications, IEEE Sens. Journ. vol. 9, n. 12, Dec 2009, pp. 1895-1906.

[8] J. Raman, E. Cretu, P. Rombouts, L. Weyten, A Closed-Loop Digitally Controlled MEMS Gyroscope With Unconstrained Sigma-Delta ForceFeedback, IEEE Sensors Journal, vol. 9, n. 3, Mar 2009 pp. 297-305.

[9] C. D. Ezekwe, B. E. Boser, A Mode-Matching Closed-Loop VibratoryGyroscope Readout Interface with a $0.004 \% \mathrm{~s} / \mathrm{JHz}$ Noise Floor over a 50 $\mathrm{Hz}$ Band, Int. Solid-State Circuit Conference (ISSCC), IEEE, 2008.

[10] A. Sharma, M. F. Zaman, F. Ayazi, A Sub-0.2\% $/ \mathrm{hr}$ Bias Drift Micromechanical Silicon Gyroscope With Automatic CMOS ModeMatching, IEEE Journ. Solid-State Circ., vol. 44, n. 5, May 2009, pp. 1593-1608.
[11] L. Prandi, C. Caminada, L. Coronato, G. Cazzaniga, F. Biganzoli, R. Antonello, R. Oboe, A Low-Power 3-Axis Digital-Output MEMS Gyroscope with Single Drive and Multiplexed Angular Rate Readout, IEEE Int. Solid-State Circuit Conf. (ISSCC) 2011, San Francisco, Feb 2011, pp. 104-106.

[12] Invensense, $M P U-9150$, Nine-Axis (Gyro + Accelerometer + Compass) MEMS Motion Tracking Device. [Online] http://invensense.com/mems/gyro/mpu9150.html, 2013.

[13] Maxim, MAX21001 Ultra-Accurate, Automotive, 3-Axis Digital Output Gyroscope, product datasheet available online at http://datasheets.maximintegrated.com/en/ds/MAX21001.pdf, 2014.

[14] STM L3G3200D MEMS motion sensor: 3-axis digital output gyroscope, product datasheet, [Online] http://www.st.com/st-web-ui/static/active /en/resource/technical/document/datasheet/DM00043564.pdf, 2014.

[15] Analog Devices, ADXRS290, Ultralow Noise, Dual-Axis MEMS Gyroscope datasheet, [online], http://www.analog.com/, 2014.

[16] M.H. Kline, Y. Yeh, B. Eminoglu, H. Najar, M. Daneman, D. A. Horsley, B. E. Boser, Quadrature FM gyroscope, Proc. of IEEE MEMS 2013, Taipei, Taiwan, Jan 2013, pp.604-608.

[17] I. I. Izyumin, M. H. Kline, Y.-C. Yeh, B. Eminoglu, C. Hyuck Ahn, V. A. Hong, Y. Yang, E. J. Ng, Thomas W. Kenny, B. E. Boser, A 7ppm, $6 \%$ hr frequency-output MEMS gyroscope, Proc. IEEE MEMS 2015, Estoril (Portugal), Jan 2015, pp. 33-36.

[18] M. H. Kline, Frequency Modulated Gyroscopes, Ph. D. final dissertation, University of California, Berkeley, Fall 2013.

[19] G. Langfelder, S. Dellea, A. Berthelot, P. Rey, A. Tocchio, A. Longoni, Analysis of Mode-Split Operation in MEMS Based on Piezoresistive Nanogauges, IEEE Journ. of Microelectromech. Syst., vol.24, n.1, pp.174,181, Feb. 2015

[20] I. Ouerghi, J. Philippe, C. Ladner, P. Scheiblin, L. Duraffourg, S. Hentz, T. Ernst, Nanowire Gauge Factor Extraction Method for Material Comparison and in-Line Monitoring, Proc. IEEE MEMS 2015, Estoril (Portugal), Jan 2015, pp. 361-364.

[21] P. Robert, V. Nguyen, S. Hentz, L. Duraffourg, G. Jourdan, and J. Arcamone, M\&NEMS: A new approach for ultra-low cost 3D inertial sensor, Proc. IEEE Sens. Conf., New Zealand, Oct 2009, pp. 963-966.

[22] J.-T. Liewald, B. Kuhlmann, T. Balslink, M. Trachtler, M. Dienger, Y. Manoli, $100 \mathrm{kHz}$ MEMS Vibratory Gyroscope, IEEE Journal of Microelectromechanical Systems, vol.22, n.5, pp. 1115-1125, Oct 2013.

[23] A. Walther, C. Le Blanc, N. Delorme, Y. Deimerly, R. Anciant, J.Willemin, Bias Contributions in a MEMS Tuning Fork Gyroscope, IEEE Journ. of Microelectromech. Syst., vol.22, n.2, Apr 2013, pp. 303.

[24] Z. Song, X. Chen, S. Huang, Y. Wang, J. Jiao, X. Li, A high-sensitivity piezoresistive gyroscope with torsional actuation and axially-stressed detection, proc. IEEE Sensors, 2003, pp.457-460, 22-24 Oct. 2003.

[25] M. S. Weinberg, A. Kourepenis, Error Sources in In-Plane Silicon Tuning-Fork MEMS Gyroscopes, Journal Of Microelectromechanical Systems, Vol. 15, No. 3, pp. 479, June 2006.

[26] A. Frangi, G. Laghi, G. Langfelder, P. Minotti, S. Zerbini, Optimization of Sensing Stators in Capacitive MEMS Operating at Resonance, IEEE Journ. of Microelectromech Syst., doi:10.1109/JMEMS.2014.2381515.

[27] ITMEMS s.r.l, MEMS Characterization Platform, Product Data-Sheet, [Online]. Available: http://www.itmems.it. Accessed Jan 2015.

[28] J. A. Geen, S. J. Sherman, J. F. Chang, S. R. Lewis, Single-Chip Surface Micromachined Integrated Gyroscope With $50 \%$ Allan Deviation, IEEE Journal of Solid-State Circuits, vol. 37, N. 12, Dec 2002, pp. 1860-1866.

[29] G. Langfelder, A. Caspani, A. Tocchio, Design Criteria of Low-Power Oscillators for Consumer-Grade MEMS Resonant Sensors, IEEE Trans. on Industrial Electronics, vol. 61, n. 1, Jan 2014, pp. 567-574.

[30] E. Tatar, S.E. Alper, T. Akin, Quadrature-Error Compensation and Corresponding Effects on the Performance of Fully Decoupled MEMS Gyroscopes, Journal Of Microelectromechanical Systems, Vol. 21, N. 3, June 2012, pp. 656-667.

[31] S. Dellea, N. Aresi, A. Longoni, Linearity of Piezoresistive NanoGauges for MEMS Sensors, Procedia Engineering 87 ( 2014 ) 1469 1472 .

[32] I.P. Prikhodko, S.A.Zotov, A. A. Trusov, A. M. Shkel, What is MEMS Gyrocompassing? Comparative Analysis of Maytagging and Carouseling, IEEE Journal of Microelectromechanical Systems, vol.22, n.6, pp.1257-1266, Dec 2013.

[33] Honeywell, Handling Sensor Bridge Offset, AN212 Sensor Products Application Note, Solid State Electronics Center, www.magneticsensors.com, accessed February 2015.

[34] W.-M. Lai, F.-M. Hsu, W.-L. Sung, R. Chen, W. Fang, Monolithic Integration of Micro Magnetic Pillar Array with Anisotropic Magneto- 
Resistive (AMR) Structure for out-of-Plane Magnetic Field Detection, Proc. IEEE MEMS 2015, Estoril (Portugal), Jan 2015, pp. 901-904.

[35] S. Dellea, G. Langfelder, A. F. Longoni, Fatigue in Nanometric SingleCrystal Silicon Layers and Beams, Journal Of Microelectromechanical Systems, in press, DOI: 10.1109/JMEMS.2014.2352792.

S. Dellea received the M.S. degree in Physics Engineering from Politecnico di Milano, Italy, in 2011, with a Thesis about fatigue effects in microscale Polysilicon. In December 2011 he won a research grant on adhesion in micro and nano devices. $\mathrm{He}$ is now attending his Ph.D., focusing on the design and test of MEMS gyroscopes based on piezoresistive NEMS gauges.

F. Giacci received the M.S. degree in Electronics Engineering from Politecnico di Milano, Italy, in 2012, with a Thesis about design of gyroscope with nanostructured sensing elements.

$\mathrm{He}$ is now attending his Ph.D., focusing on the design of characterization and operation electronics for capacitive and piezoresistive gyroscopes.

A. F. Longoni received the M.S. degree in Nuclear Engineering from Politecnico di Milano, Italy, in 1972. In this university, he is currently a Full Professor of Detectors, Microsensors and Microsystems.

His recent research is in the fields of MEMS, radiation detectors, and material analysis instrumentation. He is the author of about 180 publications and of some patents.

Prof. Longoni received the Accademia Nazionale dei Lincei Prize for novel instrumentation for cultural heritage diagnosis.

G. Langfelder received the Ph.D. degree in Information Technology in 2009 from Politecnico di Milano, Italy, where he is now an Assistant Professor of Digital Imaging.

His research focuses on CMOS sensors, MEMS, and related electronics. He is the author of about 80 refreed publications and of 4 patents. In 2014, he was a co-founder of ITmems s.r.l. Dr. Langfelder was the recipient of the Premio di Laurea Accenture in 2005 and the Premio per la Promozione della Ricerca Scientifica in 2011, granted by Rotary International. 